XXI.

Aus der II. medizinisehen Klinik der Königlichen Charité in Berlin.

\title{
Ueber die praktische Verwertung der Registrierung des Herzschalls.
}

\author{
Von \\ Stabsarzt Dr. Reinhard Ohm, \\ Assistent der Klinir.
}

(Mit 1 sbbildung und 31 Kurren im Text.)

\section{Von den bisherigen Methoden und Ergebnissen der Herzschall- registrierung.}

Von den verschiedenen Methoden der Herzschallregistrierung seien diejenigen moderneren Verfahren herrorgehoben, die als klinische Untersuchungsmethoden bekannter geworden sind.

Hinsichtlich der älteren bzw. weniger gebräuchlichen Methoden wie die von Donders, Hürthle, Holowinski, Marbe, Einthoven verweise ich auf die Zusammenstellung, die 0 . Weiss und G. Joachim in ihrer Arbeit ${ }_{n}$ Registrierung und Reproduktion mensehlicher Herztöne und Herzgeräusche $\left.{ }^{1}\right)^{4}$ gegeben haben, sowio auf die Literaturangaben in dem Buch von H. Gerhartz, Die Registrierung des Herzschalls²).

Unter den moderneren für diø Klinik in Betracht kommenden Verfahren nenne ich das von O. Frank, „Die unmittelbare Registrierung der Heratöne $\left.{ }^{3}\right)^{u}$, bei dem die Schwingungen durch Vermittlung eines mit der schallempfindlichen Platte der Brustwand aufgelegten Pbonendoskops auf oine mit einem Spiegelchen armierte Gummihaut übertragen werden.

Ein von dem Spiegelchen reflektiertes Strahlenbündel photographiert die Membranschwingungen.

Weiter erwähne ich das Verfahren von 0 . Weiss, bei dem die mit Hilfe eines Trichters von der Brustwand abgenommenen Schwingungen eine Seifenhaut erregen und durch diese auf ein Glashebelchen übertragen werden. Die Schwingungen des Hebels werden photographiert. Anstatt eines Hobels benutzt S. Garten ein im Zentrum der Seifenhaut magnetisch fixiertes Eisenstäubchen zur Registrierung ${ }^{4}$ ).

H. Gerhartz bedient sich oiner Methode, bei dor die zentralen Schwingungen einer Kollodiummembrane durch Vermittelung eines Stäb-

1) Pflüger's Arch. f. Physiol. 1908. Bd. 123.

2) Verlag ron Jul. Springer. Berlin 1911.

3) Münohener med. Wochensch. 1904. S. 953.

4) Ueber die Verwendung der Seifenmembran zur Schallregistrierung. Zeitschr. f. Biologie. 1911. Bd. 56 . 
chens auf ein Spiegelchen übertragen werden. In der bekannten Weise verzeichnet hierbei der vom Spiegel reflektierte Strahl die Schwingungen ${ }^{1}$ ).

Die bisherigen Untersuchungen über Herzschallregistrierung beziehen sich auf die Frequenz der registrierten Schwingungen, ihre zeitlichen Beziehungen zum Spitzenstoss, arteriellen Puls, Elektrokardiogramm, Venenpuls, auf die Berechnung der Dauer von Systole und Diastole und die Beziehungen der Töne zum Beginn der Systole und Diastolo [vgl. die bereits erwähnten Untersuchungen von 0 . Woiss und Joachim, H. Gerhartz, ferner A. Weber's und A. Wirth's Arbeit: nZur Registrierung der Herztöne ${ }^{4}$ nach 0 . Frank ${ }^{2}$ ), sowie meine eigenen Untersuchungen, zusammengefasst in meiner Monographie: "Venenpuls und Herzschallregistrierung als Grundlage für die Beurteilung der mechanischen Arbeitsleistung des Herzens nach oigenen Methoden $\left.{ }^{3}\right)^{\text {"] }}$. Es haben weiter 0 . Weiss und Joachim Versuche zur Reproduktion der aufgezeichneten Schwingungen in den ursprünglichen Schall unternommen (vgl. die bereits zitierte Arbeit) und dadurch die Korrektheit ihrer Kurven zu beweisen versucht. Dieses Vorgehen verliert indessen an Beweiskraft, wenn man bedenkt, dass unter Umständen schon ein Schlag gegen die Brustwand ein den registrierten Schwingungen des Tons ähnliches Bild erzeugen kann, wie A. Weber and A. Wirth (vgl. die bereits zitierte Arbeit) gezeigt haben.

Klinische Untersuchungen über die registrierten Schwingungen beim pathologischen Herzschall haben insbesondere G. Joachim in der erwähnten gemeinsamen Arbeit mit 0 . Weiss, sowie in einer späteren Arbeit der genannten Autoren: "Registrierungen von Herztönen und Herzgeräuschen beim Menschen ${ }^{4}$ ), ${ }^{,}$ferner auch H. Gerhartz (vgl. das von ihm herausgegebene schon genannte Buch) angestellt.

Auf eipige Punkte ihrer Érgebnisse möge kurz eingegangen werden.

H. Gerhartz bemüht sich den Nachweis zu erbringen, dass er mit dem von ihm benutzten Apparat reine Luftschallschwingungen, die er anscheinend mit den rom Ohr wahrgenommenen Schwingungen identifiziert, aufzeichnet. Demgegenüber muss betont werden, dass die Juftschallschwingungen, wio sie übrigens mit jedem Apparat registriert werden, Umsetzungen mechanischer Brustwandschwingungen sind. werden.

Mit den Wahrnehmungen des Ohrs können sie nicht identifiziert

Uebereinstimmend wird von allen Untersuchern die längere Dauer und grössere Unregelmässigkeit der registrierten Schwingungen bei $\mathrm{Ge}-$ räuschen betont. Das trifft auch in meinen Untersuchungen zu, wenngleich ich bemerken möchte, dass bei kurzen Geräuschen die registrierten Schwingungen nicht länger anhalten als bei Tönen, dagegen oft nur durch die grössere Schwingungsamplitude imponieren.

1) H. Gexhartz, „Die Registrierung des Herzschalls." Verlag von Julius Springer. Berlin 1911.

2) Doutsches Arch. f. klin. Mөd. 1912. Bd. 105.

3) Verlag von August Hirschwald. Berlin 1914.

4) Arch. f. klin. Med. 1910. Bd. 98. 
Was die einzelnen pathologischen Schallerscheinungen betrifft, so findet G. Joachim bei der Mitralinsuffizienz im allgemeinen eine grössere Distanz zwischen Beginn der aufgezeichneten Schwingungen und Beginn des Druckanstiegs in der Karotis als bei normalen Tönen und Karotispuls. Weiss und Joachim geben in ihrer orsten Arbeit in Pflüger's Archiv hierfür eine bestimmte Erklärung, nämlich eine Verspätung der Oeffnung der Aortenklappen infolge des gegonüber der Norm bestehenden geringeren Ventrikeldrucks, da ein Teil des Blutes durch die insuffiziente Mitralis in den Vorhof entweicht. In ihrer späteren (oben zitierten) Arbeit im Archiv für klinische Medizin führen die genannten Untersucher eine Reihe anderer Erklärungen für die Erscheinung an. Auch ich finde in meinen Untersuchungen bei Mitralinsuffizienz eine grössere Differenz zwischen Beginn der Schwingungen und dem arteriellen Pulsanstieg, erblicke aber den Grund hierfür nicht in einer Verspätung der Sprengung des Aortenklappenschlusses, sondern in einem tatsächlichen früheren Beginn der Schwingungen gegenüber der Norm. Dieser frühere Beginn ist erzeugt durch eine Mitbeteiligung des kräftiger arbeitenden Vorhofs.

G. Joachim und 0 . Weiss sprechen ebenfalls bei der Analyse der Kurven von Vorschwingungen, die der Präsystole angehören ${ }^{1}$ ).

Sie stellen den Satz auf, dass allo Schwingungen, die dem Karotispuls um weit mehr als 0,08-0,09 Sekunden vorangehen, der Präsystole angehören, also Vorhofstöne sind. Die Mitralinsuffizienz aber nehmen indessen die Untersucher von diesem Satz ausdrücklich aus.

Nach meiner Meinung sind bisher die Vorhofschwingungen bei der Registrierung viel zu wenig beachtet worden. Wie sich aus meinen eigenen Untersuchungen ergeben wird, fällt ihnen aber eine wichtige Rolle zu.

Hinsichtlich der registrierten Schwingungen bei Mitralstenose finden Weiss und Joachim in jedem einzelnen Fall eine ausserordentliche Konstanz der Formation der Schwingungen. Das Geräuschbild einer Herzaktion soll in der Kurve der der übrigen Herzaktionen gleichen.

Ich kann hierzu ergänzend betonen, dass ich in verschiedenen Fällen typischer Mitralstenose sehr ähnliche Bilder erhalte, wie meine nachfolgende Darstellung zeigen wird.

Was die Aortenstenose betrifft, so scheint mir aus den Kurven von Weiss der während der Systole anhaltende Charakter der Schwingungen herrorzugehen, was ich nach meinen Kurven völlig bestätigen kann. Dasselbe finde ich bei der Pulmonalstenose. Von diesem Vitium habe ich bei Weiss und Joachim keine Kurven gefunden; auch nicht in dem Buch von Gerhartz, dessen Kurvon übrigens den Weiss'schen sehr ähnlich sind.

Völlig stimme ich mit Weiss und Joachim und Gerhartz darin überein, dass die Registrierung im allgemeinen dio zeitlichen Verhältnisse viel oxakter zu beurteilen gestattet, als das mit der Auskultation möglich ist, wie die Registrierung überhaupt eine wertvolle Ergänzung unserer Auskultationsmethode darstellt.

1) Registrierungen von Herztönen und Herzgeräuschen beim Menschen. Arch. f. klin. Med. 1910. Bd. 98. S. 535 . 


\section{Eigene Untersuchnngen.}

Mit meinen eigenen Versuchen habe ich hauptsächlich einen praktischen Zweck verfolgt. Es sollte die registrierte Kurve mit dem auskultatorisch erhobenen Befunde verglichen, ferner geprüft werden, ob für die einzelnen dem $\mathrm{Ohr}$ sich darbietenden Schallphänomene bestimmte Formen in den Kurven sich ergeben und zutreffendenfalls, ob diese Formen bei den gleichen Schallerscheinungen stets wiederkehren.

Von Ausmessungen der Kurven habe ich bei diesen Untersuchungen abgesehen. Deutliche Unterschiede in dem Beginn der Schwingungen sowie deren Frequenz - und nur solche klar mit dem Auge zu erfassenden Unterschiede können bei der praktischen Beurteilung verwertet werden - müssen in übersichtlichen Kurven auch ohne Messungen zu erkennen sein. Zur zeitlichen Beurteilung habe ich den Radialispuls, sowie in vielen Fällen den Venenpuls mit meinen eigenen Methoden mitgezeichnet. Zahlreiche Registrierungen bei Menschenmit normalen Tönen, bei Herzkranken mit charakteristischen Herzgeräuschen oder anderen abnormen auskultatorischen Befunden usw. liegen diesen Untersuchungen zugrunde.

Bei den Vergleichen muss daran festgehalten werden, dass die mechanischen Schwingungen erst von unserm Ohr als entsprechende Schallerscheinungen perzipiert werden. Man kann aber nicht ohne weiteres sagen, dass alle während der Herzrevolution auftrctenden, rasch verlaufenden Schwingungen stets zu einer entsprechenden Schallerscheinung führen müssen. Dass in einzelnen Fällen Schwingungen besser zur Verzeichnung kommen als sie gehört werden, dafür mögen später einige Beispiele angefübrt werden.

Das aber bedeutet eine Ueberlegenheit der Registrierung vor der Auskultation.

Zur Ausarbeitung einer eigenen Methode der Registrierung des Herzschalls kam ich deshalb um ein geeignetes und dabei hochempfindliches Verfahren mit meinen Registriermethoden des Arterien- und Venenpulses zu kombinieren. Zwar war in der Verwendung der Seifenblase ein sehr empfindliches Verfahren gegeben. Ein praktischer Nachteil der Seilenhaut liegt indessen darin, dass sio für jeden Versuch neu hergestellt werden muss und nur eine kurze Lebensdauer besitzt. $\Lambda$ usserdem lässt sie sich schlecht als Spiegelmethode - wie ich sie benötigte - ausbilden. Die anderen Spiegelmethoden hingegen waren mir einerseits nicht genügend einfach, andrerseits nicht genügend empfindlich, um sie mit den mir notwendig erscheinenden Dämpfungsvorrichtungen verbinden zu können.

Ein aus einer dünnflüssigen Gelatinelösung gezogenes Häutchen nun eignete sich für meine Zwecke. Es steht an Empfindlichkeit der Seifenmembrane kaum nach und hat dabei den Vorzug unbegrenzter Haltbarkeit. Die Empfindlichkeit der Gelatinehaut gestattet die Anwendung starker Dämpfung - wie sie in verschiedener Weise die Horzschallregistrierung erfordert. Die Gelatinehaut lässt sich ferner als Spiegelmethode gut verwenden. 
Um die Ergebnisse der Herzschallregistrierung richtig einzuschätzen, muss man sich darüber klar sein, dass das, was wir registrieren einerlei welchen Apparat man immer anwenden mag - mechanische Brustwandschwingungen sind. Diese enthalten nun nicht nur die den Schall erzeugenden mechanischen Schwingungen, sondern es sind ihnen wahrscheinlich Eigenschwingungen, in dio die Brustwand durch die Herzaktion versetzt wird, beigemengt. Es ist weiterhin zu berücksichtigen, dass Elemente der Herzspitzenstossbewegung sich der Brustwand mitteilen. Sache der Aufnahmetechnik ist es, die erwähnten Entstellungen auszuschalten und möglichst nur die den Schall erzeugenden mechanischon Schwingungen $z u$ registrieren.

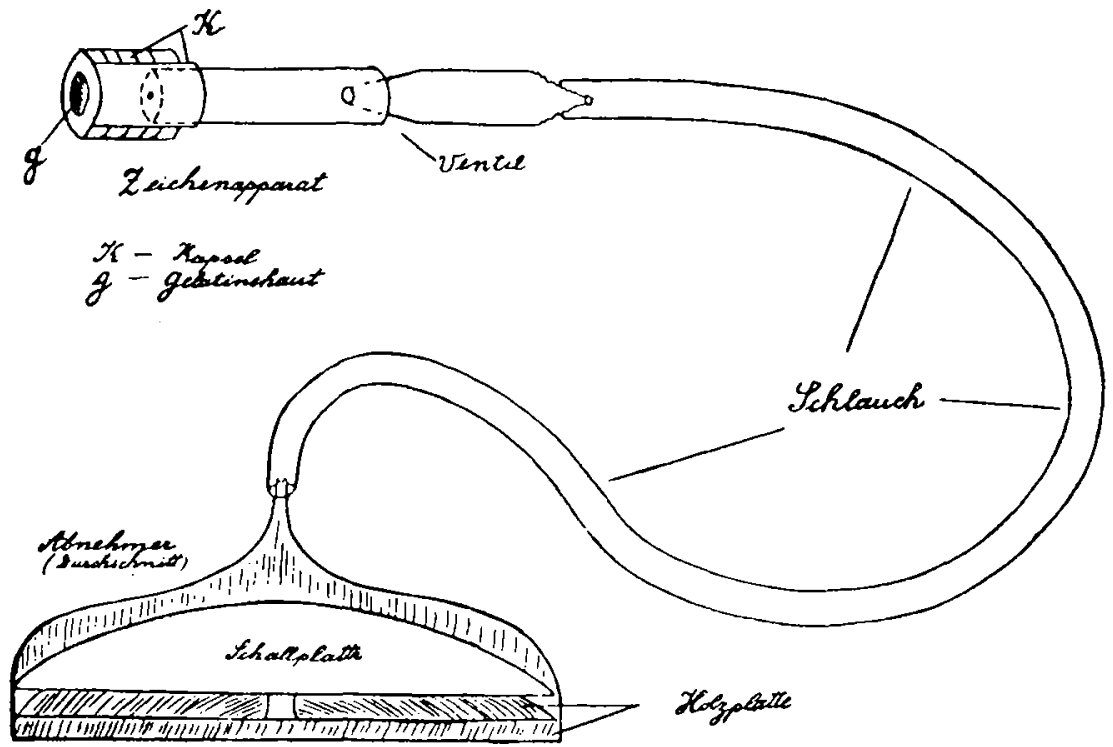

Das wird durch verschiedene Massnahmen erstrebt. Zunächst ist die Bauart des Apparates von Wichtigkeit. Weiterhin spielt die Lagerung des Patienten eine gewisse Rolle.

Hinsichtlich der Bauart des von mir konstruierten Apparates will ich unter Hinweis auf meine Arbeiten

1. die Verwendung eines Gelatinehäutchens für die Registrierung des Herzschalls ${ }^{1}$ ) und insbesondere

2. meine Honographie: Venenpuls- und Herzschallregistrierung als Grundlage für die Beurteilung der mechanischen Arbeitsleistung des Herzens nach eigenen Methoden ${ }^{2}$ )

hier nur kurz die Einrichtungen hervorheben, die die Ausmerzung bzw. Dämpfung entstellender Bewegungsvorgänge bezwecken.

1) Zeitschr. I., exper. Palh. u. Ther. 1912. Bd. 11.

2) Verlag von August Hirschwald. Borlin 1914. 
Die Spitzenstossbewegung wird bei meinem Apparat dadurch abgefangen, dass die schallempfindliche Platte der Schallabnehmevorrichtung nicht direkt der Brustwand aufliegt, sondern dass eine zweite $1 / 2 \mathrm{~cm}$ dicke undurchdrückbare starre Holzplatte vorgeschaltet ist (vgl. die Abb.).

Die grobe Bewegung des Spitzenstosses kann hier nicht durchgehen, während die rasch verlaufenden den Schall erzeugenden Schwingungen die Wand passieren. Eine derartige Vorrichtung zum Abfangen der Spitzenstossbewegung oder seiner Anteile ist notwendig. Wenn z. B. mit offenem der Gegend des Spitzenstosses aufgesetzten Trichter registriert wird, so kann man den Ablaul der Spitzenstossbewegung erhalten. Ich bringe in Kurve 1 eine solche Aufnahme, die ein Kardiogramm von auffallender Regelmässigkeit darstellt.

Eine andere, aber nicht so wirksame Möglichkeit, die grobe Bewegung des Spitzenstosses entweichen zu lassen, ist an meinem Apparat dadurch gegeben, dass das Leitungsrohr aus $z$ wei Teilen besteht, die von einander entfernt und nach Belieben genähert werden können, so dass ein in seiner Weite regulierbares aber auch vollkommen rerschliessbares Ventil entsteht (vgl. die Abb.). Ich benutze diese Einrichtung indessen zu dem besonderen $Z_{w e c k}$, dio den Schall erzeugenden Schwingungen selbst zu dämpfen und $z$ war in einem dem jeweiligen Zweck angepassten Grade.

Auf die Zwockmässigkeit dieser Massnahme kommo ich später bei der Besprechung der Kurven zurück.

Lässt sich nun die Spitzenstossbewegung des Herzens mit Sicherheit ausschalten, so gilt das nicht ohne weiteres auch für die Eigenschwingungen, in die die Brustwand durch die Herzaktion versetzt wird. Diese kann man einigermassen dadurch dämpfen, dass man bei der Registrierung eine zweckentsprechende Lagerung wählt - nämlich die Rückenlage. Hierbei sinkt das Herz etwas zurück, so dass seine Erschütterungen die Thoraxwand wenigstens nicht so unmittelbar treffen können.

Wenn also auch nicht mit absoluter Sicherheit bohauptet werden kann, dass die registrierte Kurve stets genau die mechanischen den Schall erzeugenden Schwingungen wiedergibt, so kann man doch sagen, dass die Kurve in der Hauptsache mit diesen Schwingungen übereinstimmt. Dafür sprechen die bereits vorliegenden Resultate der Herzschallregistrierung und das bestätigen meine eigenen speziellen Untersuchungen hierüber, die den Gegenstand der vorliegenden Arbeit bilden.

Bemorken will ich noch, dass Fehler von Seiten des Registrierinstrumentes selbstverständlich in die Kurve nicht hineingetragen werden dürfen. Bei der Ausarbeitung meiner Methode habe ich besonderen Wert darauf gelegt, derartige Fehler zu vermeiden. Ich verweise auf meine bereits zitierten Arbeiten und will mich hier nur darauf beschränken, zwei Bilder zu bringen, von denen das eine (Kurve 2) den Grad der Dämpfungsfähigkeit des von mir als Zeichenmembrane verwendeten hoch- 
Ueber die praktische Verwertung der Registrierung des Herzschalls. 305
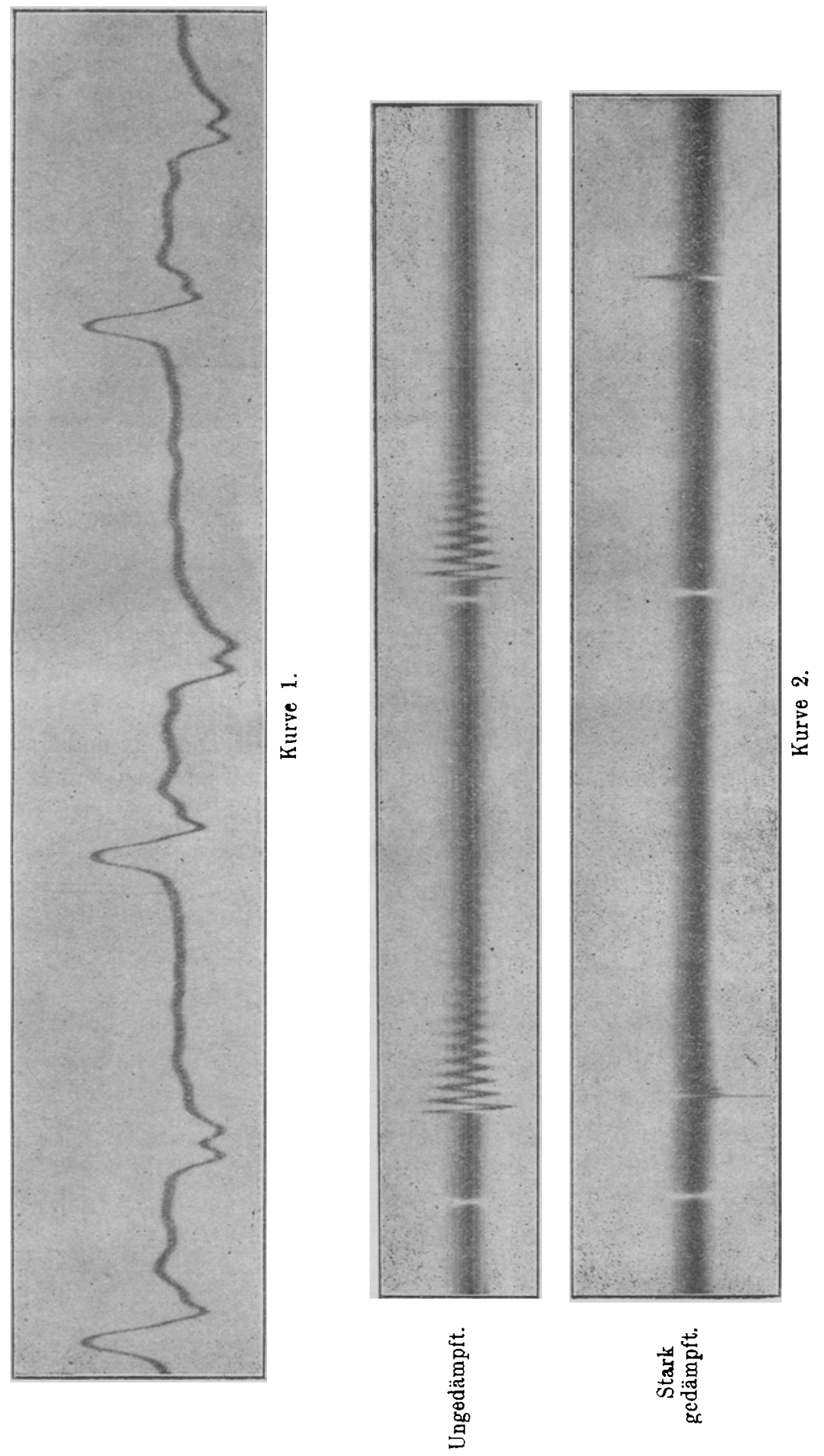
empfindlichen Gelatinehäutchens, das andere (Kurve 3) die Figenperiode des gesamten Registriersystems - erzeugt durch kurzen Anschlag gegen die Holzplatte des Schallabnehmers bei ungedämpfter Gelatinehaut darstellt.

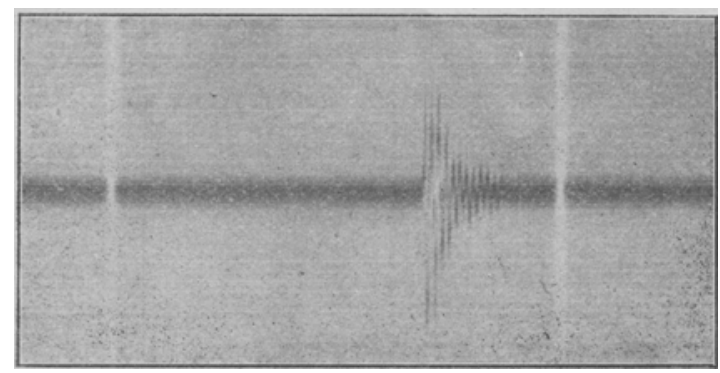

Kurve 3 .

Die Eigenschwingungszahl des gesamten Systems liegt bei über 200 in der Sekunde. Die Zeit zwischen $\mathrm{zwei}$ Schattenlinien $1 / 3$ Sekunde.

Nach diesen Vorbemerkungen will ich zu der Besprechung meiner Untersuchungen übergehen.

\section{Die registrierten Schwingungen bei reinen umgrenzten Trnen herzgesunder Menschen.}

(Hierzu Kurven 4-7.)

Bei reinen umgrenzten Tönen pflegen Austreibungszeit und Pause in der Kurve frei oder doch annähernd frei von Schwingungen zu verlaufen.

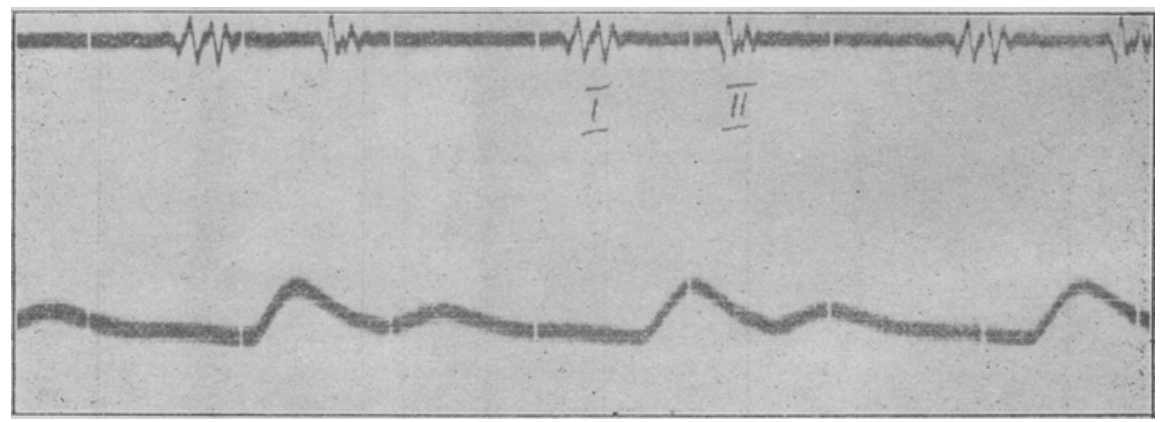

Kurve 4.

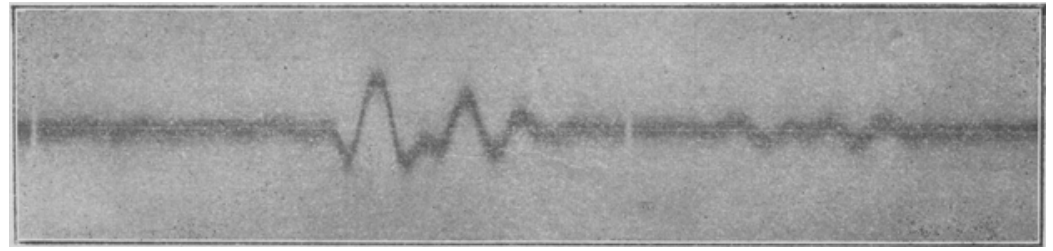

Kurve 5. 
Die Schwingungen setzen meist scharf ein und endigen ebenso, so dass Erschütterungen, die von der Tätigkeit der Vorhöfoherrühren, gewöhnlich nicht oder nur andeutungsweise $\mathrm{zu}$ sehen sind. Angedeutet sind solche Vorhofzacken z. B. in Kurve 6 .

Die zum ersten Ton gehörende Schwingung setzt sich aber stets deutlich ab. Auch in Kurve 7 , in der der Tenenpuls gleichzeitig mitgezeichnet ist, gehen kleinste, eben wahrnehmbare Zäckchen der dem ersten 'Ton entsprechenden Schwingungsgruppo voran, dio etwa mit dem Gipfel der präsystolischen Schwankung (Vorhofwolle) des Venenpulses zeitlich zusammenfallen. Es handelt sich offenbar um Schwingungen, die gegen Ende der Vorhofsystole entstehen.

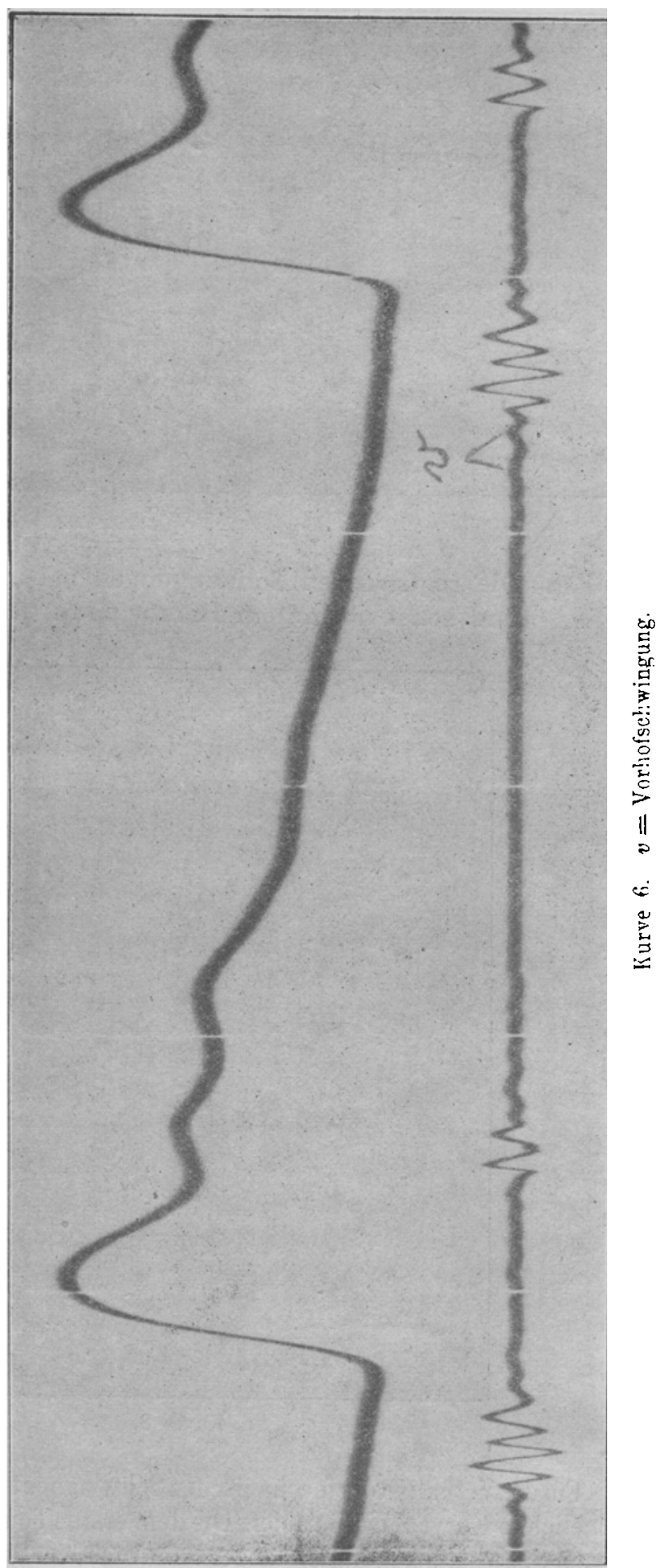




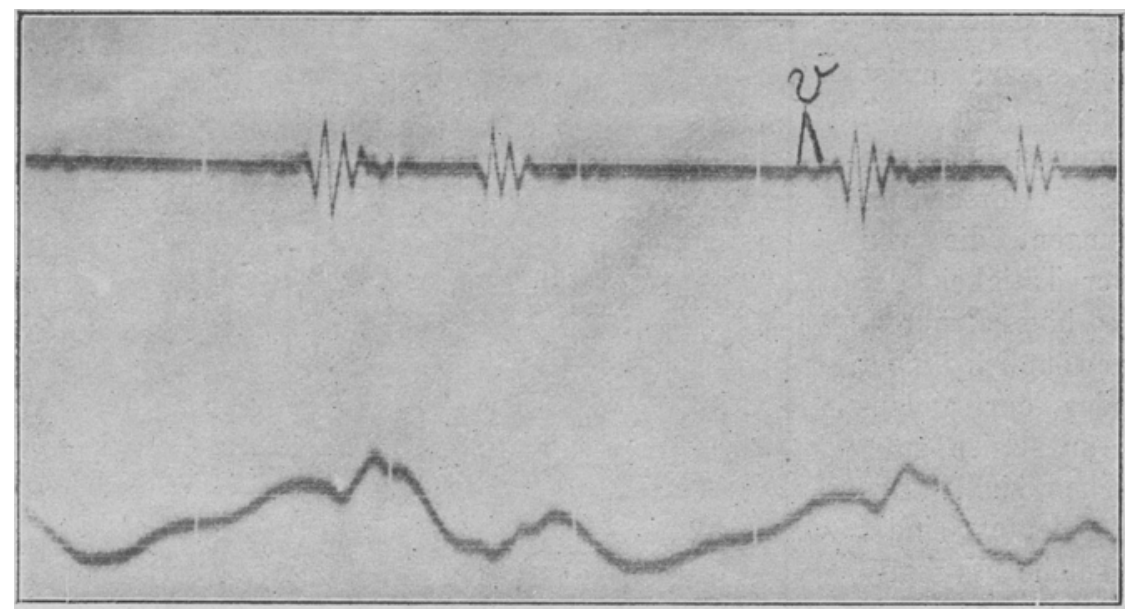

Kurve 7.

II. Die registrierten Schwingungen bei unreinem I. Ton (und sonst normalem Herzbefund). (Hierzu Kurve 8.)

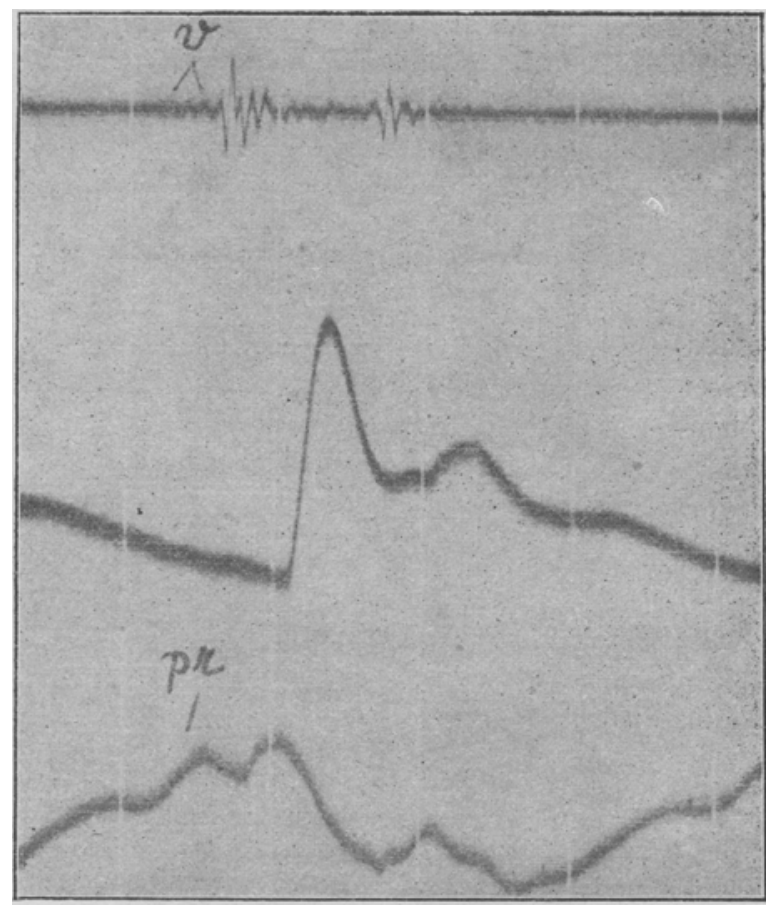

Kurve 8.

Für das Verhalten der mechanischen Schwingungen bei unreinem ersten Ton bringe ich in Kurve 8 ein Beispiel. Die dem ersten Ton entsprechenden mechanischen Schwingungen halten länger an. Auch hier gehen der dem ersten Ton entsprechenden Schwingungsgruppe kleinere Zacken voran, die mit der Vorhofschwankung des gloichzeitig mit registrierten Venenpulseskorrespondieren. 
III. Die registrierten Schwingungen bei verstärkter Vorhoftátigkeit.

a) Vorhofschwingungen bei reinen Tönen.

(Hierzu Kurven 9 und 10.)

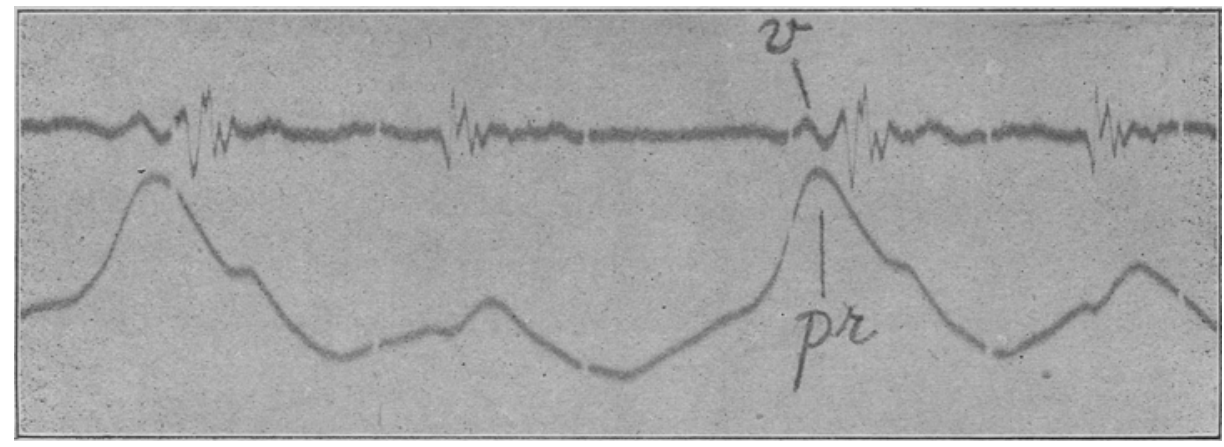

Kurve 9. $\mathrm{pr}=$ Präsystolische oder Vorhofschwankung.

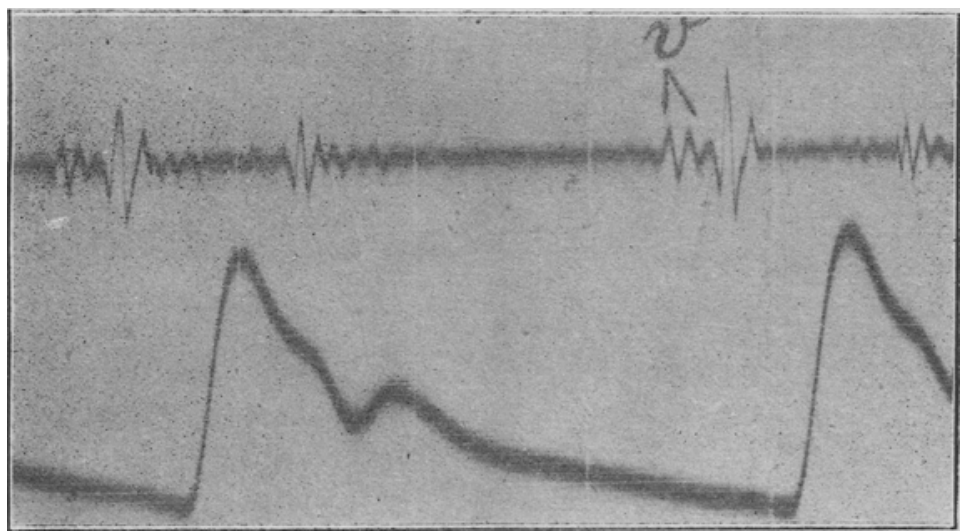

Kurve 10.

$\Lambda$ uch bei sonst reinen und normalen Tönen kann die Vorhoftätigkeit verstärktsein, was sich bei der Auskultation durchaus nicht bemerkbar zu machen braucht. Ich bringe eine Aufnahme in Kurve 9 von einem im ganzen vergrösserten Herzen nach einer schweren Diphtherie, an die sich Lähmungs-Frscheinungen anschlossen. Der Pat. befindet sich noch in klinischer Behandlung.

Es fällt in der Kurve eine kräftige mehr flache Schwingung auf, die der sich deutlich abhebenden Schwingungsgruppe des ersten Tons vorangeht. Sie kann nur als Vorhofschwingung gedeutet werden. In Uebereinstimmung damit zeigt auch der gleichzeitig mitregistrierte Venenpuls eine auffallend grosse präsystolische Schwankung Vorhofwelle). Die Töne hörten sich völlig rein an.

In einom anderon Fall mit vergrössertem Herzen aber normalem auskultatorischen Befund wurde ein Bild gewonnen, wie es Kurve 10 darstellt. Hier sind zwei scharfe Schwingungen von kleinerer Amplitude verzeichnet, die der zum ersten Ton gehörenden Schwingungsgruppe deutlich vorangehen. Schon der sehr frühe Beginn der Zacken im Vergleich zu dem mitgezeichneten Radialpuls weist darauf hin, dass die Sch wingungen von der Vorhoftätigkeitstammen. 
Roinhard Ohm,
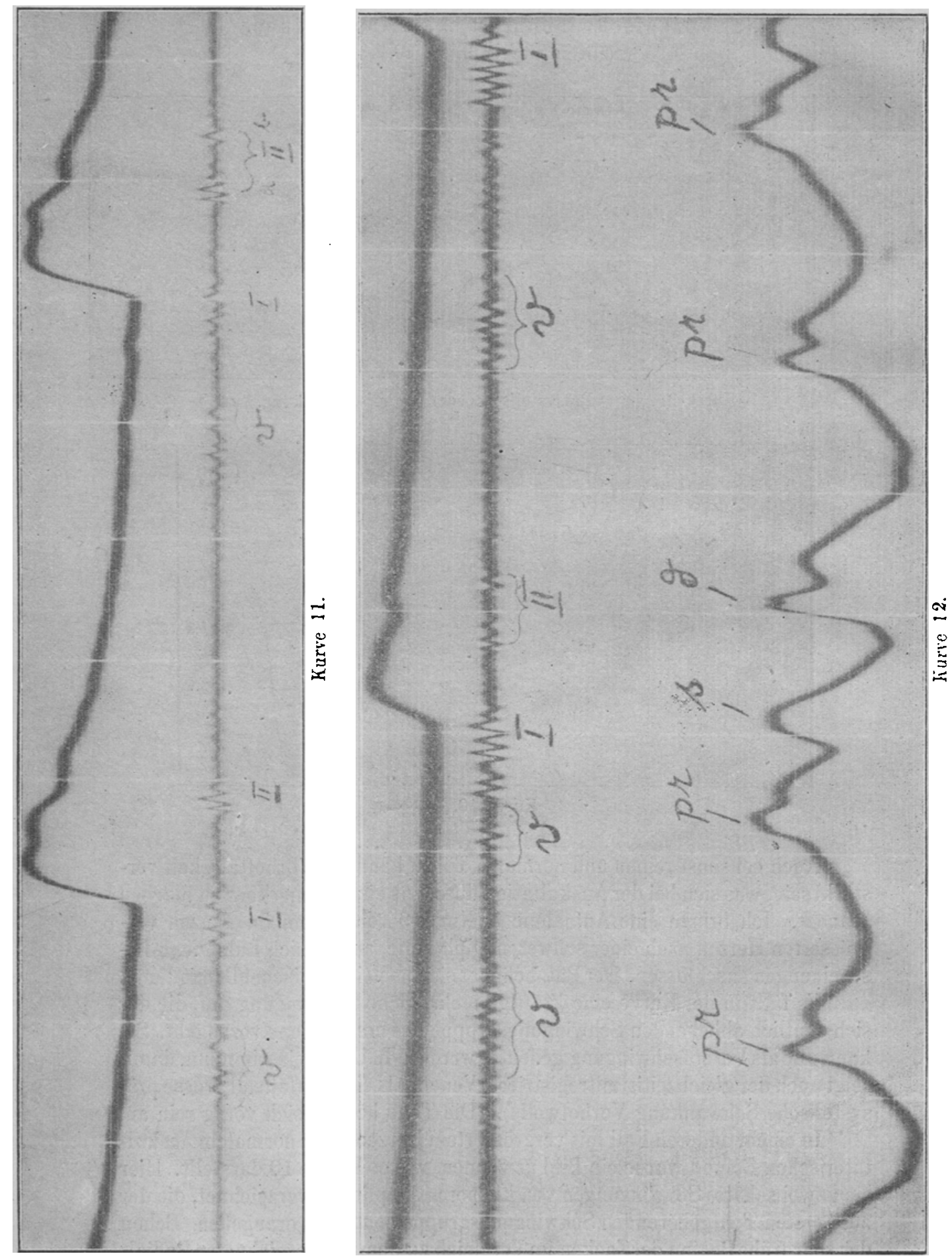
b) Vorhofschwingungen bei verstärkter isolierter Vorhoftätigkeit in einem Falle von Adams-Stocke'scher Krankheit.

(Hierzu Kurven 11-13.)

Der Patient, von dem die Bilder stammen, war vom 6. März bis 2. April 1913 in der Kinik in Behandlung. Er ist 15 Monate später seinem Leiden orlegen. Die Kurven sind in einem Anfalle von Bradykardie gezeichnet.

Man sieht in Kurve 11 vor der zum ersten Ton gehörenden Schwingungsgruppe und zwar durch eine längere Brücke schwingungsfreier Zeit von ihr getrennt die kräftigen isoliert auftretenden Vorhofschwingungen verzeichnet. Die Auskultation ergab zeitweilig ein systolisches Goräusch und zeitweilig einen gespaltenen zweiten Ton.

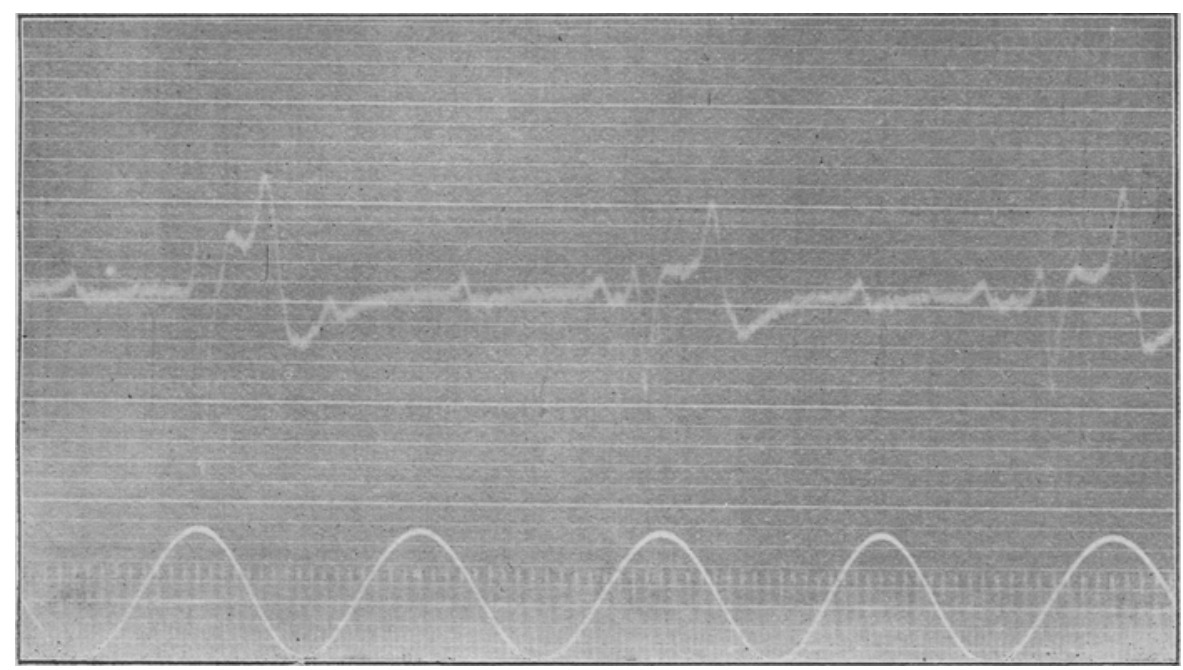

Kurve 13.

Die Vorhofschwingungen konnton mit Hilfe der Auskultation als ein eigentümliches undeutliches fernes Murmelgeräusch wahrgenommen werden. In der Kurve hingegen kommen sie sehr deutlich zum Ausdruck.

Dass es sich wirklich um Vorhofschwingungen handelt, beweist eine zweite Aufnahme (Kurve 12), in der der Venenpuls mitgezeichnet ist. Man sieht mit den isolierten Vorhofschwingungen der Tonkurve zusammenfallend die isolierte Vorhofschwankung des Venenpulses.

Das zu dem Fall gehörende Elektrokardiogramm ist in Kurve 13 beigefügt.

c) Vorhofschwingungen bei Mitralinsuffizienz.

(Hierzu Kurven 14-17.)

Die bei der Mitralinsuffizienz gesteigerte Arbeitsleistung der Vorhöfe, zunächst des linken, später auch des rechten, lässt nach den vorangegangenen Darlegungen erwarten, dass kräftigere von der Vorhoftätigkeit herrührende mechanische Schwingungen in der Kurve zur Verzeichnung kommen. 


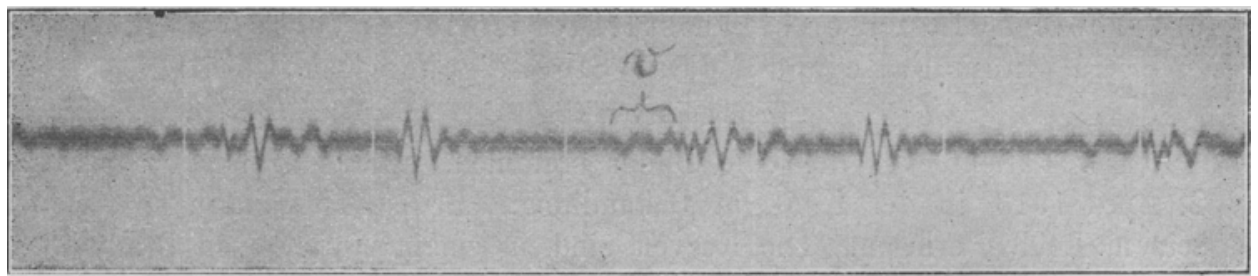

Kurve 14.

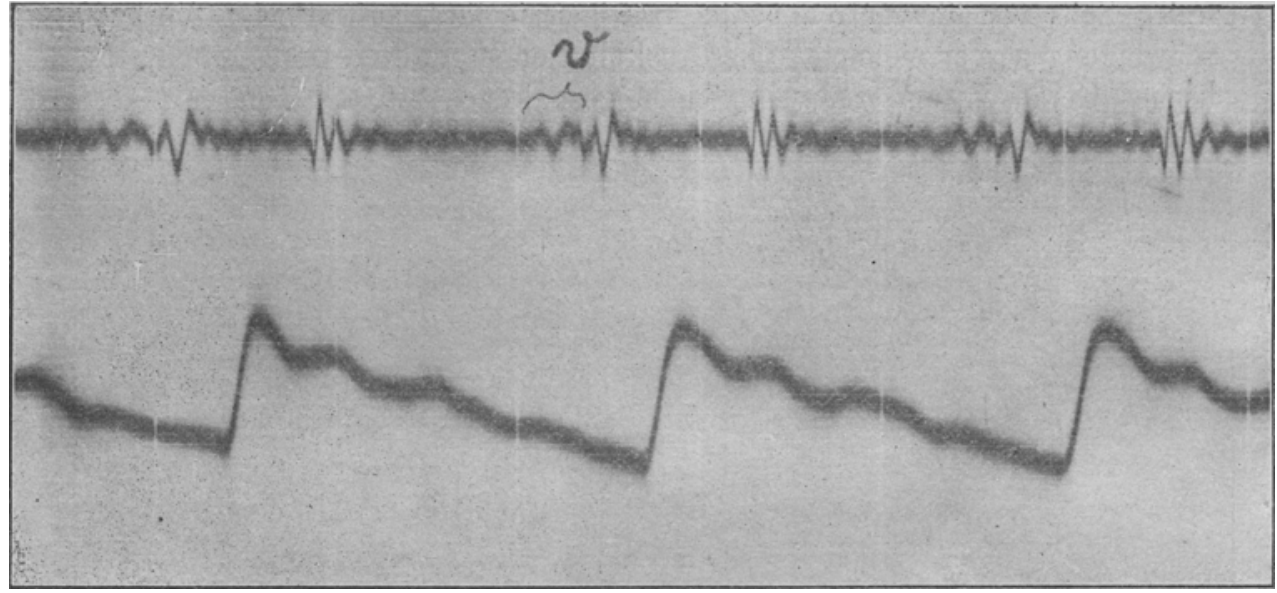

Kurve 15.

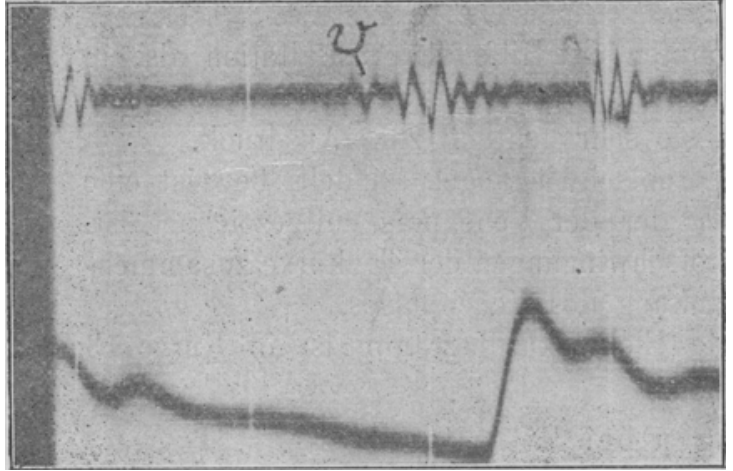

Kurve 16

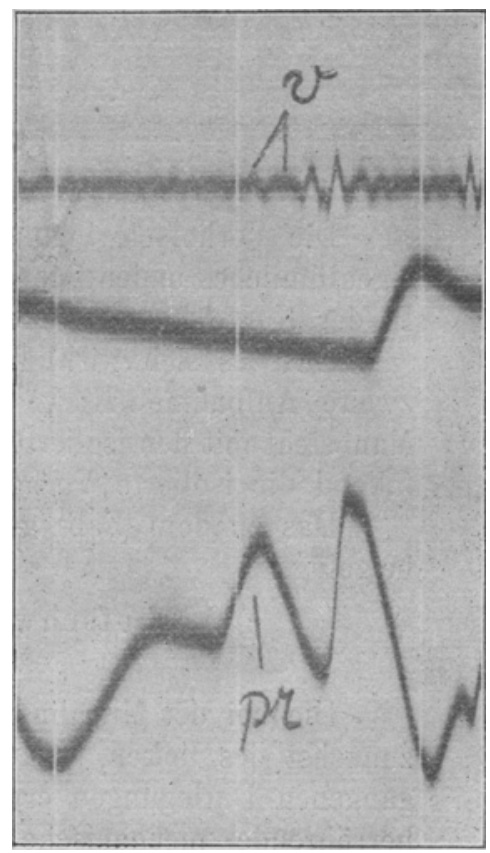

Kurve 17. 
Wir werden demnach ähnliche Bilder erhalten wie in den vorher beschriebenen Fällen der verstärkten Vorhoftätigkeit. Das Geräusch tritt dabei durch die grössere Amplitude der Schwingungen meistens auch durch eine besonders hervortretende Unregelmässigkeit derselken hervor. Das Bild der mechanischen Sehwingungen des Geräusches hat dabei den Charakter des Decrescendos.

Kurven 14-17 zeigen Beispiele verschiedener Fälle von den bei kompensierter Mitralinsuffizienz verzeichneten Schwingungen. Der Decrescendocharakter tritt überall deutlich hervor, so dass gegen Ende der Austreibungszeit ein mehr oder weniger langes schwingungsfreies Intervall entsteht. Kurven 16 und 17 stammen von demselben Patienten. In Kurve 17 ist ein stärkerer Grad der Ventildämpfung (geöffnetes Zuleitungssystem) angewandt. Die Vorhofsschwingungen sind aber auch hier gut erkennbar. Sie fallen in das Ende der präsystolischen Schwankung des gleichzeitig mitregistrierten Venenpulses.

\section{Die Kurvenform der registrierten Schwingungen bel kompensierter Mitralstenose.}

(Hierzu Kurven 18-22.)

Bei der kompeusierten Mitralstenose gelangen Schwingungsformen zur Verzeichnung, die als besouders typisch gelten können. Die Kurven 18 bis 22 entsiammen verschiedenen typischen Fällen von kompensierter Mitralstenose mit kräftigem Crescendogeräusch und paukendem ersten Ton.

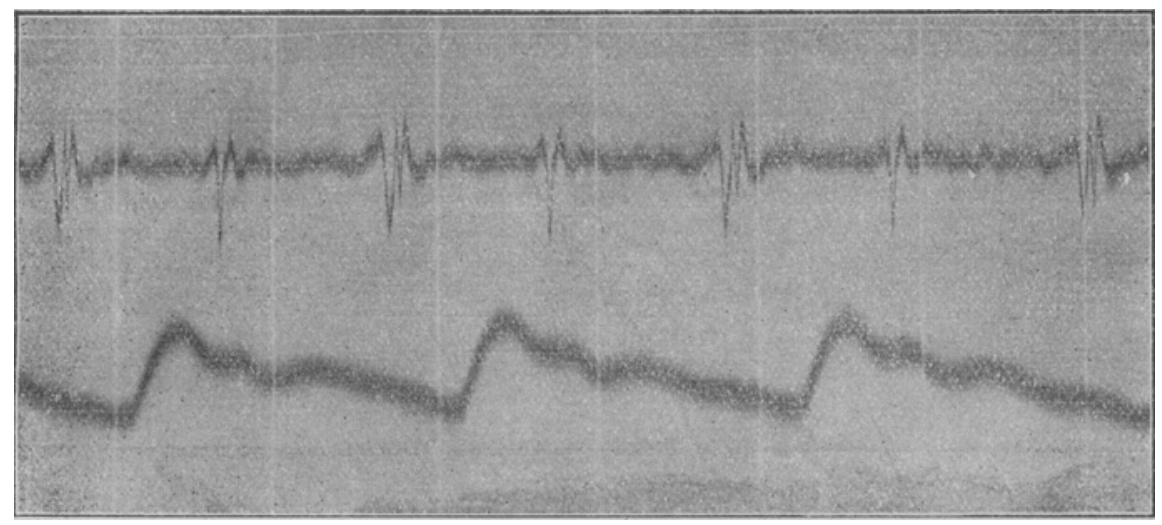

Kurve 18.

Man erkennt leicht die grosse Lebereinstimmung der Kurvenbilder. Es ist zunächst ein mehr oder weniger träger Anstieg bemerkbar, der dem Anschwellen des Crescendos entspricht; darauf folgen die dem Schnapp entsprechenden kräftigen Schwingungen von dem charakteristischen Aussehen, wie es die Bilder zeigen.

In den meisten Kurven ist gleichzeitig auch ein diastolisches Geräusch zur Darstellung gelangt.

In Kurve 21 sieht man neben dem trägen, dem Crescendo entsprechenden Anstieg noch einige dem Schnapp vorangehende Schwingungen. 


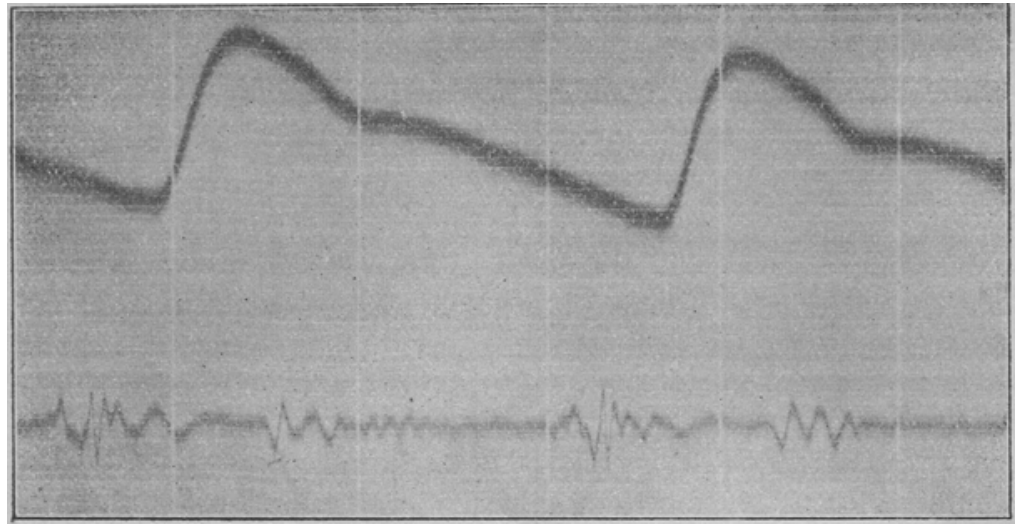

Kurve 19.

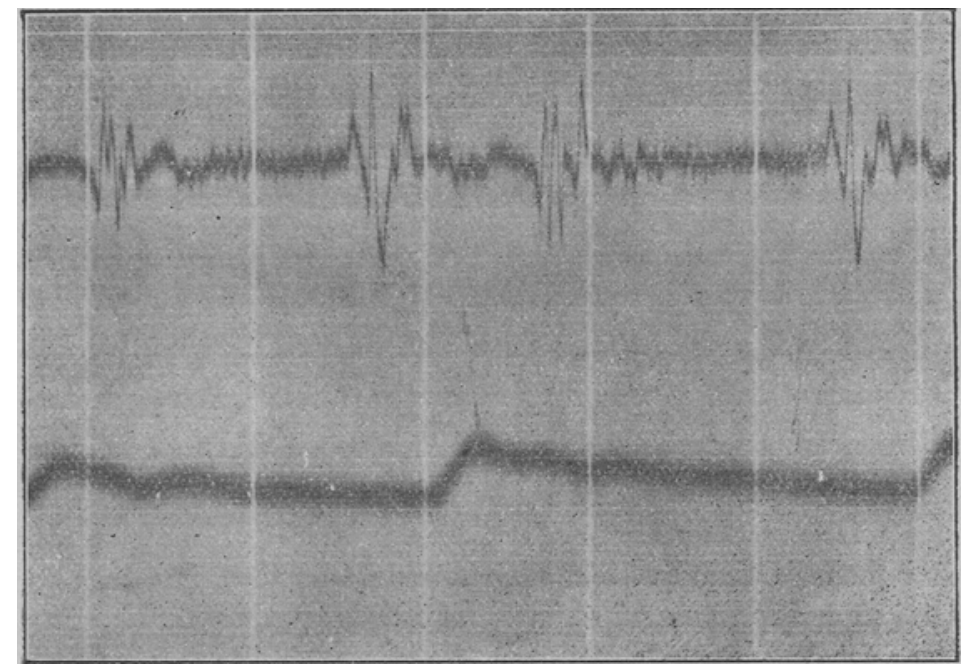

Kurve 20

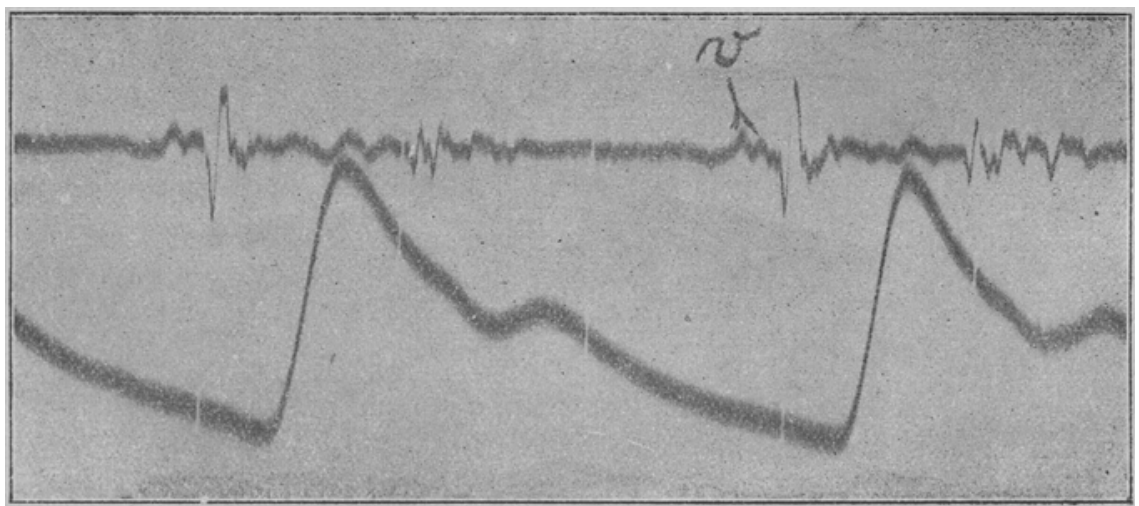

Kurve 21. 


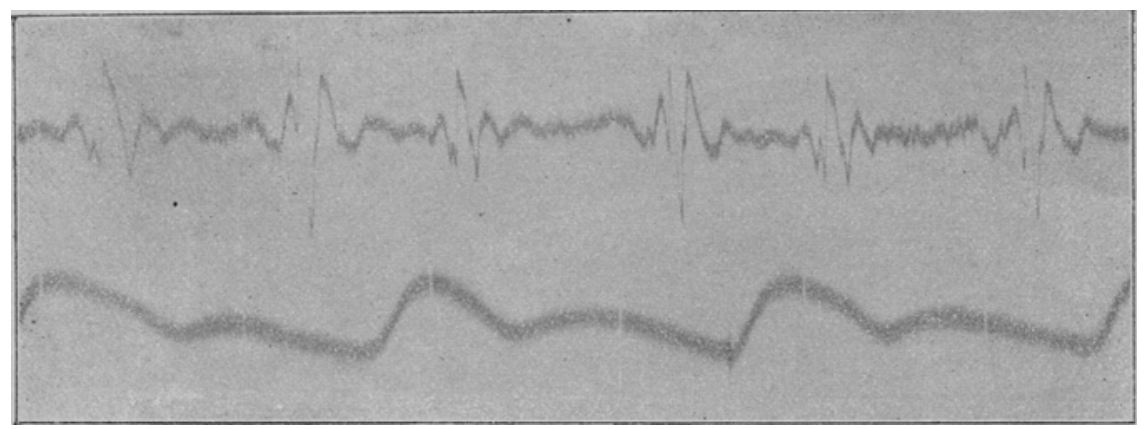

Kurve 22.

V. Die Formen der mechanischen Schwingungen bei den systolischen Stenosengerăuschen.

(Hierzu Kurven 23-27.)

a) Bei der Aortenstenose.

Ich bringe als Beispiel einen typischen Fall dieses Klappenfehlers in drei Aufnahmen bei verschiedenen Graden der Ventildämpfung.

In Kurve 23 ist das Zuleitungsrohr völlig geschlossen. Die Schwingungen sind von grosser kräftiger Anplitude und füllen die Austreibungszeit vollständig aus. In Kurve 24 ist bei der Aufnahme das Zuleitungsrohr wenig, in Kurve 25 mehr geöffnet. Trotz der Dämpfung bleibt der Charakter der mechanischen Schwingungen, die während dor ganzen Systole anhalten, gewahrt. Diese Feststellung ist wichtig gegenüber den Schwingungen bei dem systolischen Geräusch der Mitralmsuffizienz, die, wie erwähnt, den Charakter des Decrescendos haben, d. h. die Schwingungen nebmen mit zunehmender Austreibung ab. Durch Anwendung der Ventildämpfung lassen sich diese Dinge gerade bei der Registrierung gut unterscheiden.

\section{b) Bei dor Pulmonalstenose.}

Das von der Aortenstenose Gesagte gilt in analoger Weise auch für die Pulmonalstenose. Auch hier füllen die mechanischen Schwingungen die Austreibungsperiode völlig aus. Kurven 26 und 27 stellen Beispiele verschiedener Fälle von Pulmonalstenose dar. Sie sind beide bei mässiger Ventildämpfung aufgenommen. Trotz dor Dämpfung sieht man die ganze Systole von Schwingungen ausgefüllt.

Bemerkenswert in den Kurven bei Pulmonalstenose ist ferner das deutliche Vorhandensein verstärkter Vorhofschwingungen. Es ist nach dem früher Gesagten nicht schwer diese kleinen aber sehr deatlich sich markierenden Schwingungen als Vorhofschwingungen $z u$ erkennen. Es handelt sich wohl um Schwingungen des rechten Vorhofs, der ja bei der Pulmonalstenose schon frühzeitig gesteigerte Arbeit zu leisten hat.

Ebenso wie bei der Aorten- und Pulmonalstenose verhalten sich die mechanischen Schwingungen nach meinen Registrieruntersuchungen beim. Septumdefekt. 


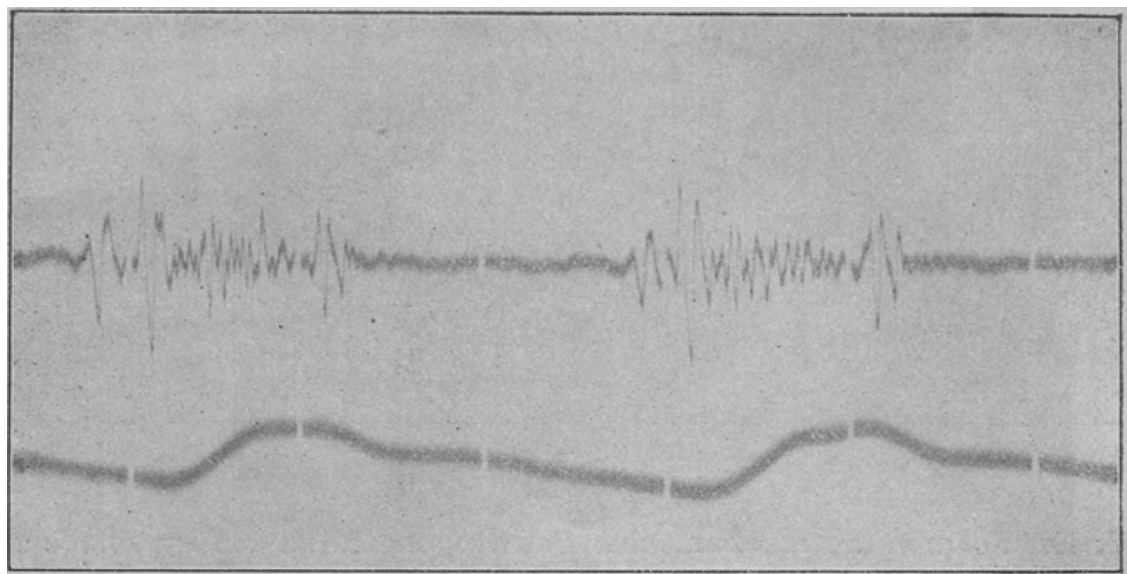

Kurve 23.

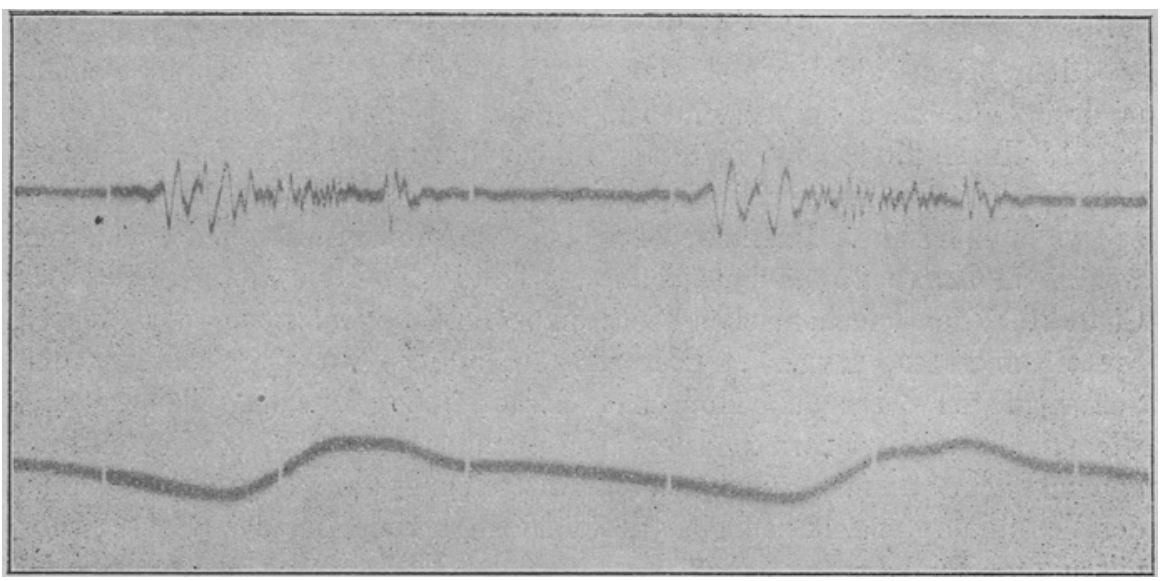

Kurve 24.

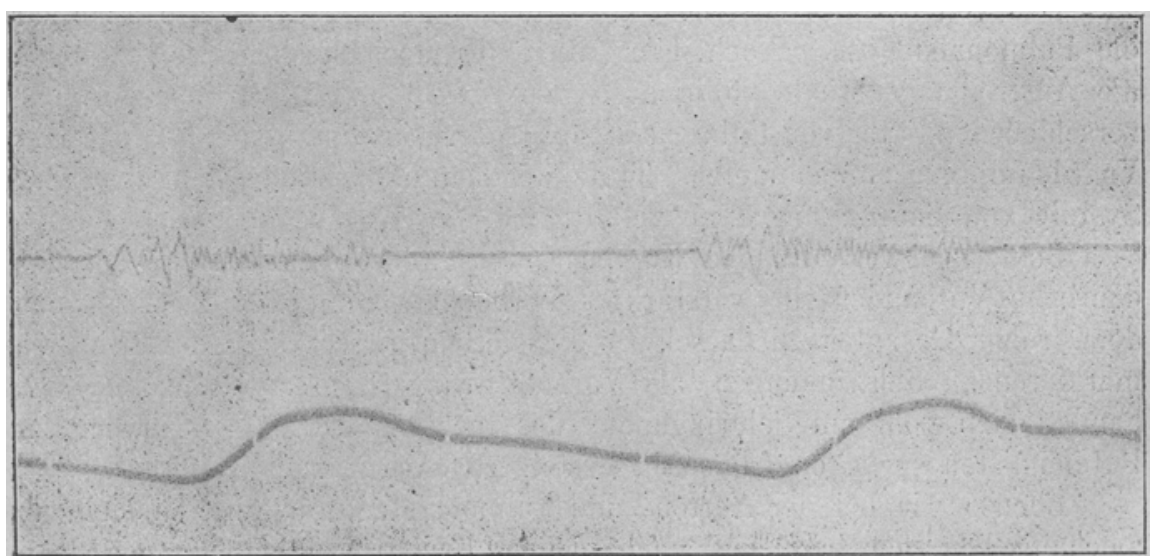

Kurve 25. 
Ueber die praktische Verwertung der Registrierung des Herzschalls. 317

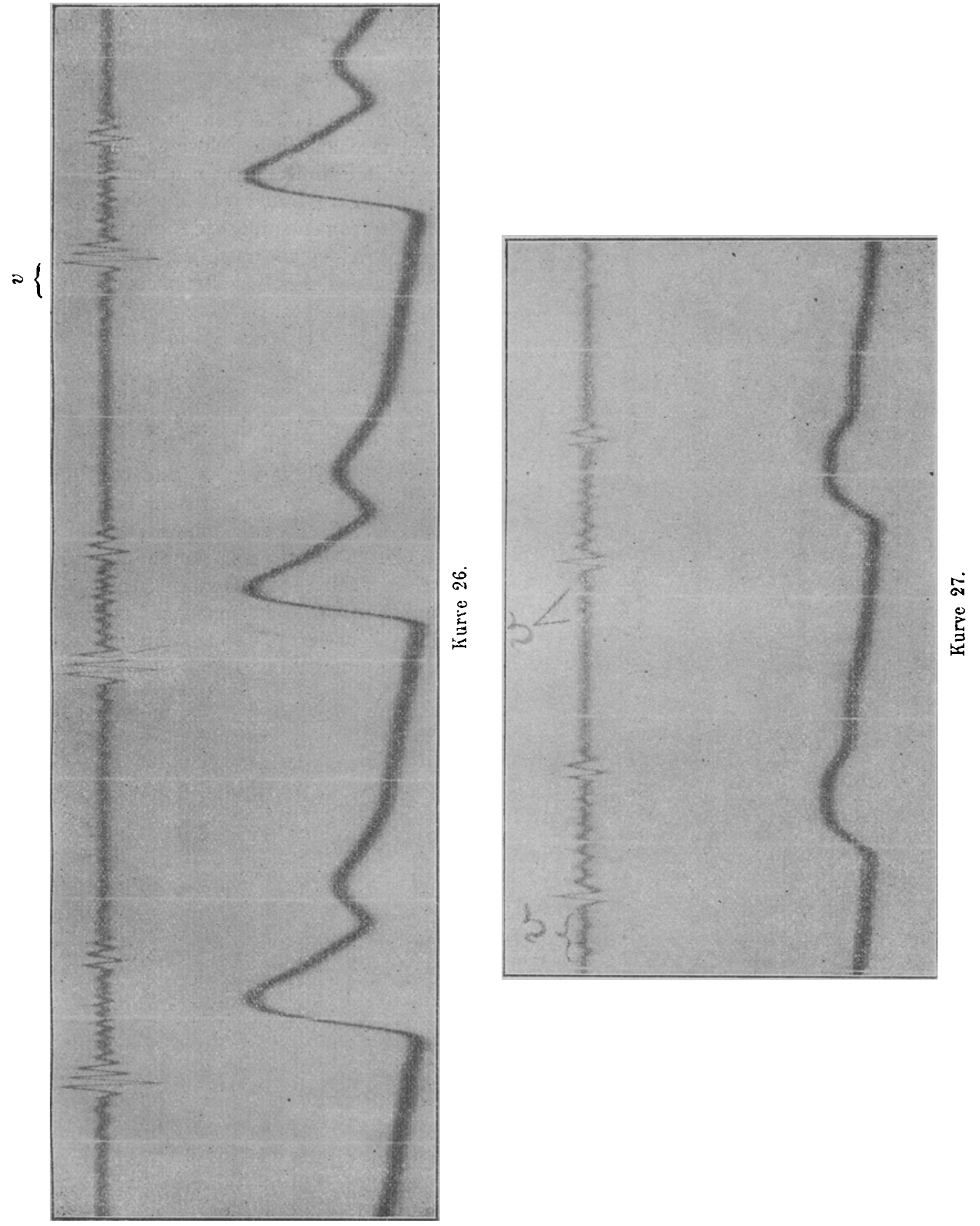


VI. Die registrierten Schwingungen bei dem diastolischen Geräusch der Aorteninsuffizienz.

(Hierza Karven 28-31.)

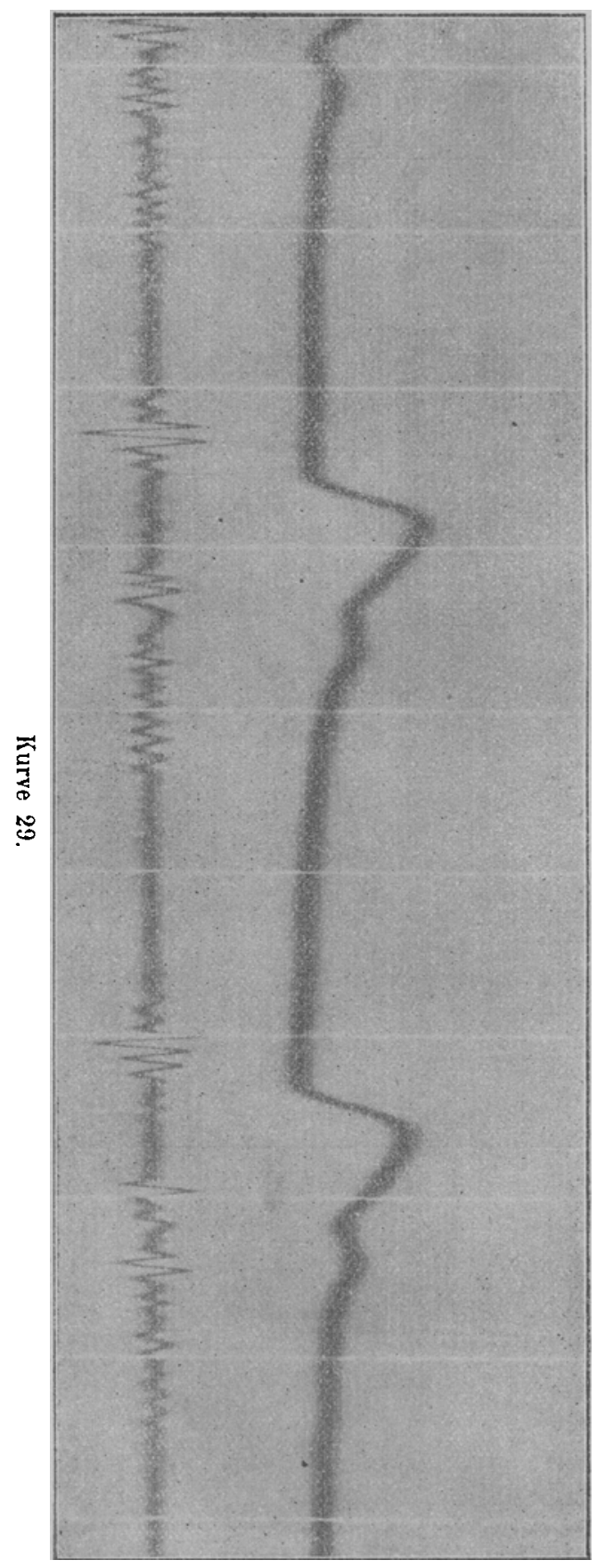

Die dem Geräusch entsprechenden mechanischen Schwingungen imponieren im allgemeinen durch ihre längere Dauer als die dem zweiten Ton entsprechende Schwingungsgruppe, wie auch die 4 von verschiedenen Fällen stammenden Beispiele zeigen.

Oft ist der zweite Ton durch ein Geräusch ersetzt. Die zum zweiten Ton gehörenden Schwingungen heben sich dann in der Kurve nicht $a b$. Ein Beispiel hierfür ist Kurve 28. In anderen Fällen sind die zum zweiten Ton gehörenden Schwingungen als solche neben den sich daran anschliessenden dem Geräusch ontsprechenden Schwingungen gut zu erkennen. Beispielo hierfür sind Kurven 29, 30 und 31 .

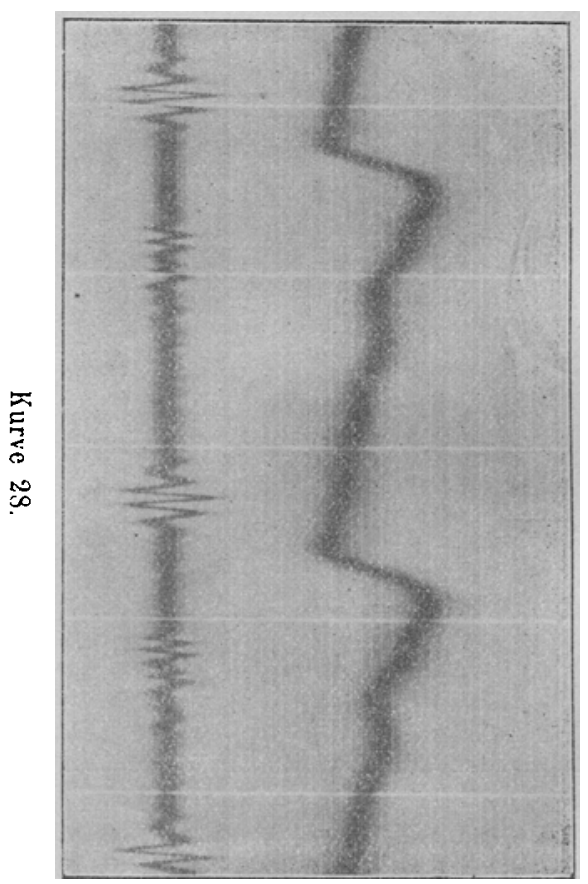




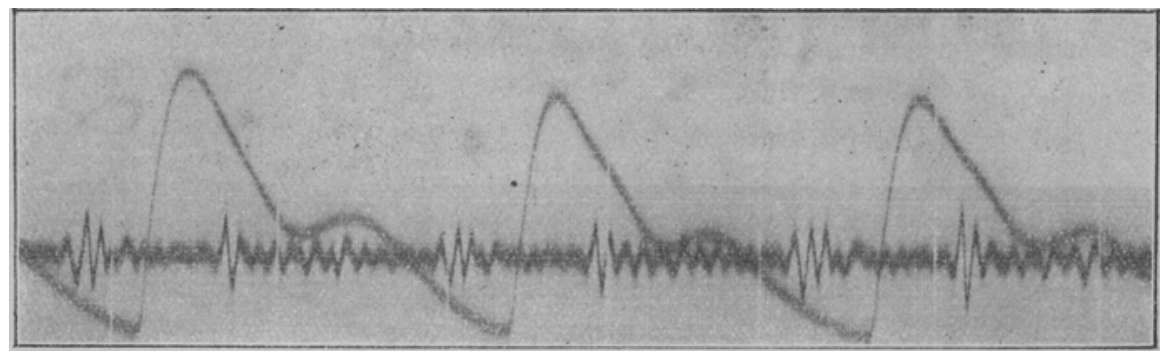

Kurve 30.

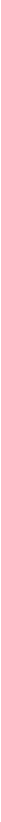

Kurve 31 .

Besonders bemerkenswert in dieser Bezjehung ist Kurve 31. Man sieht hier nicht nur deutlich die den zweiten Ton erzeugende kurze Schwingungsgruppe, sondern es liegt sogar zwischen ihr und dem nachfolgenden Geräusch ein schwingungsireies Intervall.

Hier ermöglicht die Registrierung überhaupt erst eine genauere Lokalisation des Geräusches wie es mit Hilfe der Auskultation nicht möglich ist.

\section{Zusammenfassung.}

Zusammenfassend ist zu sagen:

Die Registrierung der mechanischen, den Herzschall erzeugenden Schwingungen ist im allgemeinen crgiebiger als die Auskultation.

Es lassen sich

1. Schwingungen zur sichtbaren Darstellung bringen, die als Schallerscheinung entweder garnicht oder doch nur undeutlich wahrgenommen werden können - nämlich die Vorhofschwingungen; 
320 R. Ohm, Ueber die praktische Verwertung der Registrierung des Herzschalls.

2. einzelne Schallerscheinungen mit Hilfe der Registrierung der entsprechenden mechanischen Schwingungen genauer in die Phasen der Herzrevolution zeitlich lokalisieren;

3. mit Hilfe der Registrierung Bilder gewinnen, die sowohl der Zeitdauer der Schwingungen wie ihrer Form nach für dic typischen Vitien charakteristisch sind.

Das gilt insbesondere für die systolischen Stenosengeräusche, für die Mitralinsuffizienz- und die Mitralstenosengeräusche.

Hinsichtlich der Bedeutung der Herzschallregistrierung zur Ermöglichung der Analyse der Venenpulskurve usw. verweise ich auf meine früheren Arbeiten. 